\title{
АНАЛИЗ И РАСЧЕТ ПАРАМЕТРОВ ДИФФУЗИИ В ДВУХКОМПОНЕНТНЫХ МНОГОФАЗНЫХ СИСТЕМАХ ПРИ «ОГРАНИЧЕННОМ» ПОСТУПЛЕНИИ КОМПОНЕНТА С МЕНЬШЕЙ ТЕМПЕРАТУРОЙ ПЛАВЛЕНИЯ
}

\author{
(C) 2018 Л. А. Молохина ${ }^{1}$, С. А. Филин ${ }^{2}$ \\ ${ }^{1}$ ул. Кибальчича, д. 2, корп. 4, кв. 86, 129164 Москва, Россия \\ e-mail: lara.molokhina@mail.ru \\ ${ }^{2}$ Российский экономический университет им. Г. В. Плеханова, Стремянный переулок, 36, 117997 Москва, Россия \\ e-mail:Filin.SA@rea.ru
}

Поступила в редакцию 31.08 .2018

\begin{abstract}
Аннотация. Целью исследования является выявление особенностей расчёта параметров диффузии в двухкомпонентных многофазных системах при «ограниченном» поступлении компонента с меньшей температурой плавления. Предложен способ решения задачи об изменении длительности инкубационных периодов для фаз, которые должны появиться в диффузионной зоне после истощения слоя данного компонента. Разработаны методики расчёта параметров диффузии в двухкомпонентных многофазных системах при «ограниченном» поступлении компонента с меньшей температурой плавления.
\end{abstract}

Ключевые слова: диффузия, фаза, межфазная граница, многофазная система, изотермический отжиг, математическая модель, компонент.

DOI: https://doi.org/10.17308/kcmf.2018.20/637

\section{ВВЕДЕНИЕ}

В технологических процессах с использованием диффузии (например, диффузионные пайка, сварка, нанесение диффузионных покрытий), при которых толщина слоя компонента А (с меньшей температурой плавления) много меньше толщины слоя компонента В, компонент А в чистом виде может полностью исчезать в процессе диффузионного отжига. В этом случае рост слоёв всех последующих фаз, которые еще не появились в диффузионном слое к моменту истощения слоя компонента А, будет происходить уже только за счёт растворения предыдущих фаз, имеющихся в диффузионном слое к данному моменту времени.

Цель исследования - предложить способ решения задачи об изменении длительности инкубационных периодов для фаз, которые должны появиться в диффузионной зоне после истощения слоя компонента с меньшей температурой плавления.

\section{ТЕОРЕТИЧЕСКИЙ АНАЛИЗ}

Данная статья является продолжением разработки феноменологической математической модели формирования и роста фаз в двухкомпонентной многофазной системе с учётом анализа факторов, влияющих на процесс диффузии в двухкомпонентной системе при неограниченном поступлении компонентов, изложенной в работах $[1,2]$. При разработке данной модели использовали следующие допущения: 1) фазы в диффузионном слое появляются в порядке, соответствующем их расположению на равновесной диаграмме состояния со стороны компонента A, и 2) появлению каждой новой фазы предшествует инкубационный период, в течение которого происходит накопление атомами на границе раздела компонентов А и В энергии, достаточной для фазового перехода.

Проблема описания роста прослойки новой фазы на начальных стадиях в общей постановке остается нерешенной и актуальной [3]. Экспериментальные и теоретические исследования процесса диффузионного взаимодействия и роста фаз следует отнести к классу реакций, для которых характерным является двухстадийность процесса образования прочных связей между атомами взаимодействующих веществ с последующим образованием диффузионной зоны. В течение первой стадии происходит образование физического кон- 
такта, т. е. осуществляется сближение соединяемых веществ на расстояния, требуемые для подготовки поверхности к такому взаимодействию. В течение второй стадии (уже химического взаимодействия) начинается рост фаз за счёт гетерогенной реактивной диффузии. При этом, если температура процесса взаимодействия выше температуры плавления одного из компонентов, начало второй стадии сопровождается частичным растворением твердого компонента в жидком (например, процессы пайки). При температуре процесса взаимодействия ниже температуры плавления компонентов стадия растворения отсутствует (например, процессы диффузионной сварки разнородных материалов). В каждом из этих случаев для образования соединения требуется определенное время, обусловленное процессами развития физического контакта и химического взаимодействия фаз - инкубационный период [3-9].

Инкубационный период без учёта возможных поверхностных явлений можно рассматривать как время жизни атома перед потенциальным барьером на границе раздела или как период времени задержки роста фаз [3-6]. Рост толщины интерметаллидной прослойки хорошо согласуются с известной степенной зависимостью [5]:

$$
h^{2}=K\left(\tau-\tau_{\text {лI }}\right),
$$

где $h-$ толщина прослойки, мкм; $\tau$ и $\tau_{\text {л }}-$ соответственно время отжига и латентный период образования интерметаллидов, ч; $K-$ постоянный коэффициент для данной температуры отжига.

Протекание процессов взаимодействия различных типов в контакте соединяемых материалов требует определенной величины энергии для активации состояния поверхностей. Эта энергия может сообщаться в виде теплоты (термическая активация), энергии упругопластической деформации (механическая активация), электронного, ионного и других видов облучения (радиационная активация) и т. д.

Для ориентировочных оценок инкубационного периода можно принять допущение, что величина межфазной энергии в случае взаимодействия двух конденсированных фаз изменяется (уменьшается) по экспоненциальному закону [7-9]:

$$
\tau_{i}^{u}=\tau_{0 i}^{u} \cdot \exp \left(\frac{E_{b i}}{2 k T}\right)
$$

где $\tau_{0 i}^{u}-$ предэкспоненциальный множитель; $E_{b i}$ - величина межфазного потенциального барьера на границе раздела фазы « $(i-1)$ - компонент В».

В работах [10-15] дана оценка длительности указанных процессов и сопоставлены результаты расчёта с имеющимися опытными данными. Эти процессы рассмотрены как для пайки, сварки плавлением, так и для сварки в твердом состоянии. Однако в данных работах рассмотрены длительности инкубационных периодов только для однофазных слоев и не рассмотрены вопросы, связанные с расчётом длительности инкубационных периодов для каждой фазы в многофазной диффузионной зоне.

В работах $[1,2]$ рассмотрены вопросы роста фаз многофазных слоев и показано, что появлению каждой новой фазы в диффузионном слое от начала процесса предшествует суммарный инкубационный период $\left(\tau_{i}^{d}\right)$, отсчитываемый от начала изотермического отжига до момента появления данной фазы и состоящий из суммы всех инкубационных периодов, предшествующих появлению данной фазы и всех предшествующих фаз (рис. 1):

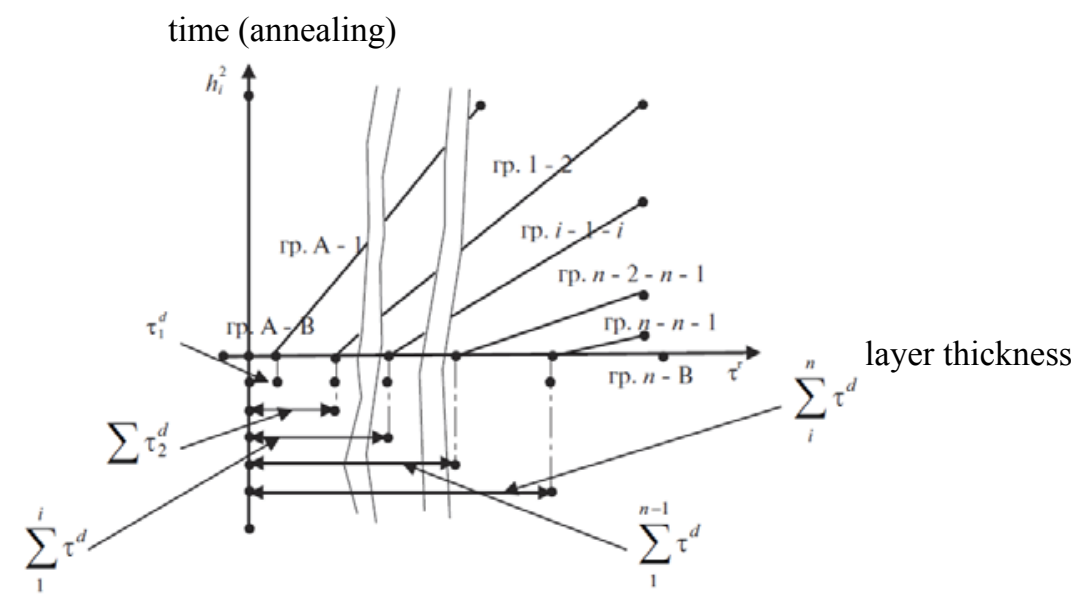

Рис. 1. График зависимости толщины слоя компонента А, пошедшего на рост фаз $i$ и $(i+1)$, от времени диффузии [Fig. 1. The schedule of dependence of thickness of a layer of the component A which has gone for growth of phases $i$ and $(i+1)$ from diffusion time] 


$$
\sum_{i=1}^{d} \tau^{d}=\tau_{1}^{d}+\tau_{2}^{d}+\ldots+\tau_{i}^{d}
$$

где $\tau^{d}$ - инкубационный период, предшествующий появлению фазы $i$ и отсчитываемый от момента появление фазы $(i-1) ; \sum_{i=1}^{d} \tau^{d}-$ суммарный инкубационный период, предшествующий появлению фазы $i$ и отсчитываемый от начала изотермического отжига.

Рост толщины слоя $\left(x_{i}\right)$ для $\boldsymbol{i}$-й фазы при этом равен:

$x_{i}=\sqrt{A_{i}\left(\tau^{d}-\sum_{i=1}^{u} \tau^{u}\right)}-\frac{C_{i+1}}{C_{i}} \sqrt{A_{i+1}\left(\tau^{d}-\sum_{i=1}^{u} \tau^{u+1}\right)}$,

где $x_{i}$ - толщина слоя фазы $i ; A_{i}$ - коэффициент скорости роста фазы $i ; A_{i+1}$ - коэффициент скорости роста фазы $(i+1) ; C_{i}-$ средняя атомарная концентрация компонента А в фазе $i ; C_{i+1}$ - средняя атомарная концентрация компонента А в фазе $(i+1) ; \sum_{i=1}^{u} \tau^{u}-$ суммарный инкубационный период, предшествующий появлению фазы $i$ и отсчитываемый от начала изотермического отжига; $\sum_{i=1}^{u} \tau^{u+1}-$ суммарный инкубационный период, предшествующий появлению фазы $(i+1)$ и отсчитываемый от начала изотермического отжига; $\tau^{d}$ - время изотермического отжига (время диффузии).

В то же время количество компонента А, перешедшее каждую соответствующую межфазную границу, подчиняется параболической зависимости от времени изотермического отжига с учётом суммарных инкубационных периодов:

$$
\sum_{i=1}^{n} h_{i}=\sqrt{B_{i}\left(\tau^{d}-\sum_{i=1}^{u} \tau^{u}\right)}
$$

где $\sum_{i=1}^{n} h_{i}$ - толщина слоя компонента А, использованного на рост всех $n$-фаз, образовавшихся в двухкомпонентной системе к соответствующему времени отжига; $B_{i}$ - коэффициент скорости перехода компонента А через каждую межфазную границу, равный тангенсу угла $\beta_{i}$ наклона прямой зависимости $h^{2}=f(\tau) ; \sum_{i=1}^{u} \tau^{u}-$ суммарный инкубационный период, предшествующий появлению фазы $i$ и отсчитываемый от начала изотермического отжига; $\tau^{d}$ - время изотермического отжига (время диффузии).
Параметры $x_{i}, C_{i}, B_{i}$ и $A_{i}$ связаны между собой (без учёта влияния на толщину слоев разницы в плотности упаковки атомов в кристаллических решетках как самих исходных компонентов, так и всех фаз двухкомпонентной системы) следующими соотношениями:

$$
\begin{aligned}
& x_{i} C_{i}=h_{i}, \\
& B_{i}=A_{i} C_{i}^{2},
\end{aligned}
$$

При этом предполагается, что длительность инкубационных периодов для каждой фазы и исследуемой температуры является величиной постоянной. Однако при изучении взаимной диффузии в системе «медь-галлий», а также в тонких пленках [16-18], установлено, что инкубационные периоды фаз, появляющихся в диффузионной зоне после истощения компонента А, уменьшаются.

Для разработки методики расчёта инкубационных периодов в двухкомпонентных многофазных системах при «ограниченном» поступлении компонента с меньшей температурой плавления проанализируем рост фаз между компонентами А (компонент с меньшей температурой плавления) и В (компонент с большей температурой плавления) при толщине слоя А, много меньшей толщины слоя В, в период времени изотермического отжига, когда компонент А полностью израсходован на рост слоев в диффузионной зоне (рис. 2 ).

Движущей силой диффузионного процесса является разность термодинамических потенциалов [17], появляющаяся в момент образования контакта на границе раздела между компонентами А и В, и далее на всех новых границах, появляющихся в моменты начала роста новых фаз. При этом величина разности термодинамических потенциалов имеет своё определенное значение для каждой из имеющихся границ раздела. В периоды стабильного роста фаз, когда в диффузионной зоне не происходит появление новых фаз, для всех существующих на данный момент границ при данной температуре отжига устанавливается определенное равновесие соответствующих разностей термодинамических потенциалов. Соответственно этому устанавливается и определенный энергетический баланс двухкомпонентной многофазной системы, связывающий между собой и поддерживающий в заданном состоянии все узловые точки на рис. 2.

В период времени, когда $\left(\tau^{d}<\sum_{i=1}^{u+1} \tau^{u}\right)$ и когда фаза $(i+1)$ еще не появилась в диффузионном слое, энергетическое состояние границы раздела «фаза $i$ - компонент В» имеет локальную неста- 


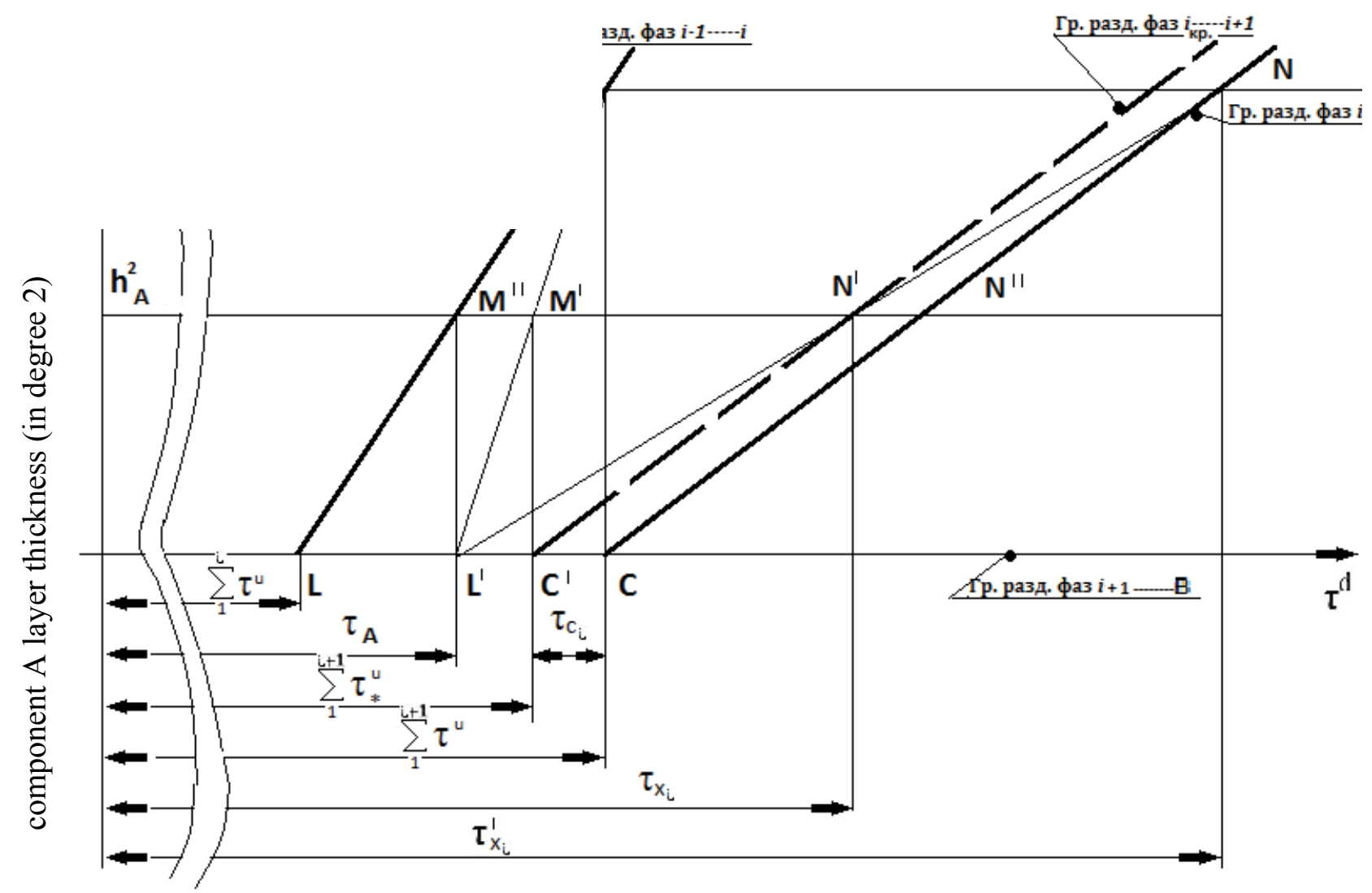

Рис. 2. Схема движения компонента А через межфазные границы при взаимной диффузии в двухкомпонентных многофазных системах

[Fig. 2. The scheme of the movement of a component A through interphase borders at interdiffusion in two-component multiphase systems]

бильность. На этой границе раздела происходит накопление энергии для начала роста фазы $(i+1)$. В момент времени $\tau_{A}$, когда чистый компонент $\mathrm{A}$ полностью израсходован, исчезает граница раздела «компонент A - фаза 1» и происходит нарушение энергетического баланса двухкомпонентной многофазной системы. Этот состояние неустойчиво, и двухкомпонентная многофазная система стремится вернуть устойчивое состояние. Путем перераспределения вещества компонентов А и В многофазная система будет стремиться к достижению в ней минимума свободной энергии Гиббса посредством снижения энергии, необходимой для начала роста фазы $(i+1)$, на той границе, на которой имеется локальное нестабильное состояние, а именно: на границе раздела «фаза $i-$ компонент В».

На рис. 2 представлены графики функций:

$$
\left(\sum_{n}^{i} h_{i}\right)^{2}=f\left(\tau^{d}\right) \text { и }\left(\sum_{n}^{i} h_{i+1}\right)^{2}=f\left(\tau^{d}\right) \text {, }
$$

где $h_{i}$ - толщина слоя компонента А, израсходованная на рост фазы $i$ и всех предшествующих фаз; $h_{i+1}$ - толщина слоя компонента А, израсходованная на рост фазы $(i+1)$ и всех предшествующих фаз; $\tau^{d}-$ время диффузии; $h_{\text {А }_{\text {кр }}}$ - толщина слоя компонента А, расходуемого на рост фазы $i$ и всех предшествующих фаз при времени диффузии $\tau^{d}=\sum_{i=1}^{u+1} \tau^{u} ; h_{\mathrm{A}}$ - толщина слоя компонента А, pacходуемого на рост фазы $i$ и всех предшествующих фаз при времени диффузии $\tau^{d}=\tau_{\mathrm{A}}$;

Анализ графиков на рис. 2 позволяет предложить следующее графическое решение задачи:

1) точки $\mathrm{M}$ и $\mathrm{N}$ пересечений с прямыми LM и $\mathrm{CN}$ функций:

$$
\begin{gathered}
\left(h_{i}\right)^{2}=f(\tau), \\
\left(h_{i+1}\right)^{2}=f(\tau),
\end{gathered}
$$

определяют толщину слоя компонента $\mathrm{A}\left(h_{i}\right.$ и $\left.h_{i+1}\right)$, пошедшего на рост фазы $i$ и $(i+1)$ к периодам 
в ремени отжига $\left(\sum_{i}^{u} \tau^{u}<\tau^{d_{1}} \leq \sum_{i+1}^{u} \tau^{u}\right)$ и $\left(\sum_{i+1}^{u} \tau^{u}<\tau^{d_{2}} \leq \sum_{i+2}^{u} \tau^{u}\right)$

2) точка $\mathrm{M}^{\prime \prime}$ пересечения прямой, параллельной оси абсцисс $\mathrm{M}^{\prime \prime} \mathrm{N}^{\prime \prime}$ с прямой функции (9), определяет толщину слоя компонента $\mathrm{A}\left(h_{\mathrm{A}}\right)$, пошедшего на рост фазы $i$, в период $\tau^{d}=\tau_{\mathrm{A}}$;

3) точки L и C пересечения оси координат $\tau^{d}$ с прямыми LM и CN функций $(2,3)$ определяют суммарные инкубационные периоды $\sum_{i}^{u} \tau^{u}$ и $\sum_{i+1}^{u} \tau^{u}$ фаз $i$ и $(i+1)$;

4) точка $L^{\prime}$ определяет начало момента изменения $\sum_{i+1}^{u} \tau^{u}$ при истощении компонента А;

5) прямая $\mathrm{C}^{\prime} \mathrm{N}^{\prime}$ параллельна $\mathrm{CN}$ и соответствует функции (8). Вследствие уменьшения необходимой энергии активации процесса для начала роста фазы $(i+1)$ после истощения компонента А происходит перемещение прямой $\mathrm{C}^{\prime} \mathrm{N}^{\prime}$ вдоль оси абсцисс до ее пересечения в точке $\mathrm{N}^{\prime}$ с прямой $\mathrm{M}^{\prime \prime} \mathrm{N}^{\prime \prime}$, параллельной оси абсцисс. Прямая $\mathrm{M}^{\prime \prime} \mathrm{N}^{\prime \prime}$ соответствует количеству компонента А, участвующему в данном процессе;

6) точка $\mathrm{C}^{\prime}$ при этом определяет длительность измененного инкубационного периода $\sum_{i+1}^{u} \tau^{u_{*}}$ (выделено квадратом на рис. 2) для фазы $(i+1)$.

Точки M, N, L и C связаны между собой энергетическим балансом, характеризующим равномерное движение компонентов и установившимся в двухкомпонентной многофазной системе в период времени $\left(\tau^{d}<\tau_{\mathrm{A}}\right)$. Точки $\mathrm{M}^{\prime \prime}, \mathrm{N}, \mathrm{L}^{\prime \prime}$ и $\mathrm{C}^{\prime}$ связаны между собой новым энергетическим балансом, который устанавливается в двухкомпонентной многофазной системе при истощении компонента А в период времени $\left(\tau^{d}>\tau_{\mathrm{A}}\right)$.

Анализ полученных соотношений в подобных треугольниках $\mathrm{MCN}$ и $\mathrm{M}^{\prime} \mathrm{C}^{\prime} \mathrm{N}^{\prime}$ позволяет записать систему уравнений для квадрата толщины $\left(h_{A}\right)$ истощающегося слоя компонента А:

$$
\left\{\begin{array}{l}
h_{A}^{2}=B_{i+1}\left(\tau_{x i}-\sum_{1}^{u+1} \tau^{u_{*}}\right), \\
h_{A}^{2}=K\left(\tau_{x i}-\tau_{A}\right), \\
h_{A}^{2}=B_{i}\left(\tau_{A}-\sum_{1}^{u} \tau^{u}\right),
\end{array}\right.
$$

где $\tau_{A}$ - время полного растворения компонента А, если $(i=1)$, или время растворения фазы $(i-1)$ при толщине слоя компонента А, равной $h_{A} ; \sum_{1}^{u+1} \tau^{u_{*}}$ 一 измененный суммарный инкубационный период для фазы $(i+1) ; K$ - тангенс угла наклона прямой $\mathrm{N}^{\prime} \mathrm{L}^{\prime}$ к оси абсцисс; $B_{i}$ и $B_{i+1}$ - коэффициенты скорости перехода компонента А через каждую межфазную границу, равные тангенсу угла $\beta_{i}$ наклона линии зависимости $h^{2}=f(\tau)$;

а также систему уравнений для определения критической толщины слоя компонента А для начала изменения инкубационного периода появления фазы $(i+1)$ в диффузионной зоне:

$$
\left\{\begin{array}{l}
h_{\mathrm{A} к р}^{2}=B_{i+1}\left(\tau_{x i^{\prime}}-\sum_{1}^{u+1} \tau^{u}\right), \\
h_{\text {Акр }}^{2}=K\left(\tau_{x i^{\prime}}-\tau_{A}\right), \\
h_{\text {Акр }}^{2}=B_{i} \sum_{1}^{u+1} \tau^{u},
\end{array}\right.
$$

где $\tau_{x i}$ - время полного растворения фазы $i$ при росте фазы $(i+1)$ и толщине слоя компонента $\mathrm{A}$, равной $h_{\text {Акр }} ; h_{\text {Акр }}-$ начальная толщина слоя компонента A, которая в процессе диффузии полностью расходуется на рост фаз от 1 до $i$ за период времени $\tau^{d}$, меньший, чем $\sum_{1}^{u+1} \tau^{u}$ (на рис. 2 определяется пересечением прямой MN с осью ординат).

Решая совместно системы уравнений (10-12) и (13-15), получим:

$$
\begin{gathered}
\sum_{1}^{u+1} \tau^{u_{*}}=\sum_{1}^{u} \tau^{u}+h_{A}^{2}\left(\frac{1}{B_{i}}+\frac{1}{K}+\frac{1}{B_{i+1}}\right), \\
K=B_{i}\left(\tau_{i+1}\right)^{u}\left(\left(\tau_{i+1}\right)^{u}+\frac{B_{i}}{B_{i+1}}\left(\tau_{i+1}\right)^{u}-\frac{h_{A}^{2}}{B_{i}}\right)^{-1},
\end{gathered}
$$

Подставив выражение (17) в выражение (16), запишем уравнение зависимости инкубационного периода появления фазы $(i+1)$ от толщины слоя компонента A:

$$
\sum_{1}^{i+1} \tau^{u_{*}}=\sum_{1}^{u} \tau^{u_{*}}+h_{A}^{2}\left(\frac{2}{B_{i}}-\frac{h_{A}^{2}}{B_{i}^{2} \tau_{i+1}^{u}}\right)
$$

где $\sum_{1}^{u} \tau^{u_{*}}$ - измененный инкубационный период фазы $i$, если $h_{\mathrm{A} i}<h_{\text {Аікр }}$.

Как видно из уравнения (15), в двухкомпонентной многофазной системе для каждой фазы $(i+1)$ существует критическое значение толщины слоя компонента А, при меньшем значении которого и 
длительности процесса отжига $\left(\tau^{d}>\sum_{1}^{i+1} \tau^{u_{*}}\right)$ инкубационный период этих фаз будет изменяться согласно уравнению (18). Изменение инкубационного периода фазы $(i+1)$ произойдет независимо от того, что предшествовало этому: истощение компонента А или же, если оно произошло значительно ранее при ( $\left.\tau^{d}>\sum_{1}^{i} \tau^{u}\right)$, растворение одной из предыдущих $(i-1)$ фаз.

Предложенное продолжение разработки модели расчетов, показанной в статьях $[1,2]$, позволяет до проведения подтверждающих экспериментов произвести теоретический расчет диффузионной зоны для случаев истощения компонента с меньшей температурой плавления в период изотермической выдержки. Также могут быть проведены и прогностические расчеты для изменения диффузионной зоны в период эксплуатации изделий при повышенных температурах.

\section{ЭКСПЕРИМЕНТАЛЬНАЯ ЧАСТЬ}

При исследовании диффузионного взаимодействия и роста фаз в системе «медь-галлий» и использовании в процессе отжига тонких слоев галлия ( $\leq 20$ мкм) применительно к разработке технологии диффузионной пайки и нанесения на поверхность изделий интерметаллических покрытий при одинаковых температурах и времени отжига фазовый состав сильно зависит от начальной толщины слоя галлия. Зависимость микроструктуры и фазового состава паяных швов меди галлием для интерметаллических фаз $\gamma_{1}, \gamma_{2}, \gamma_{3}$ и $\xi_{1}$ при одинаковых температуре и времени выдержки изотермического отжига от толщины исходного количества галлия представлена на рис. 3 .

Исследования микроструктуры и фазового состава паяного шва показали, что время появления $\xi_{1}$-фазы в паяном шве при одинаковых температуре и времени изотермического отжига в первую очередь зависит от исходной толщины слоя галлия.

На базе предложенной теоретической методики учёта влияния на длительность инкубационных периодов момента истощения прослойки компонента с меньшей температурой плавления (представлена на рис. 3) были проведены эксперименты для подтверждения возможности использования данной теоретической методики.

На основе результатов, полученных при исследовании взаимодействия в системе «медь-галлий» при изотермическом отжиге, и условии, что галлий не истощается в период проведения экспериментов, рассчитаны параметры диффузии в данной системе. Результаты исследований и расчётов представлены на рис. 4-7.

Исследования роста фаз в системе «медь-галлий» на первом этапе проводили на образцах раз-

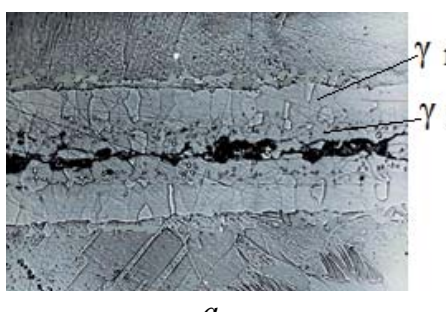

concentration (atomic) of gallium in a layer

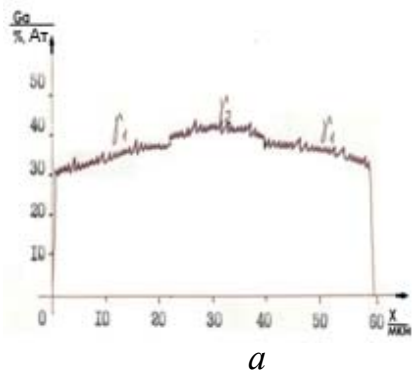

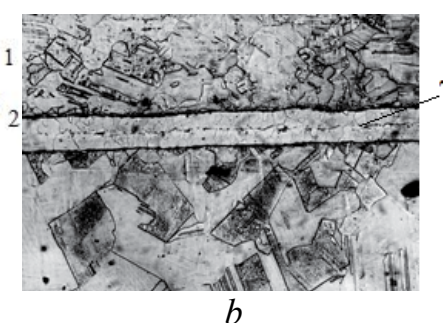

$b$

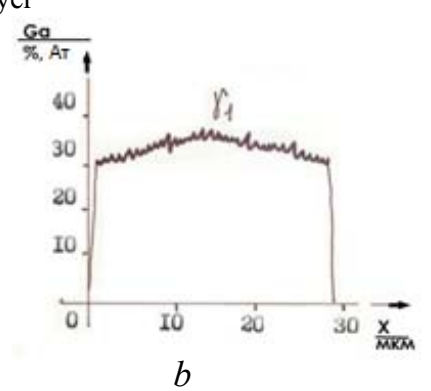

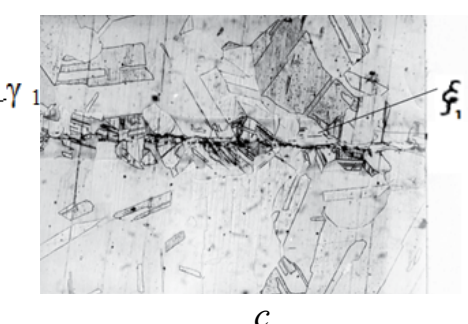

$c$

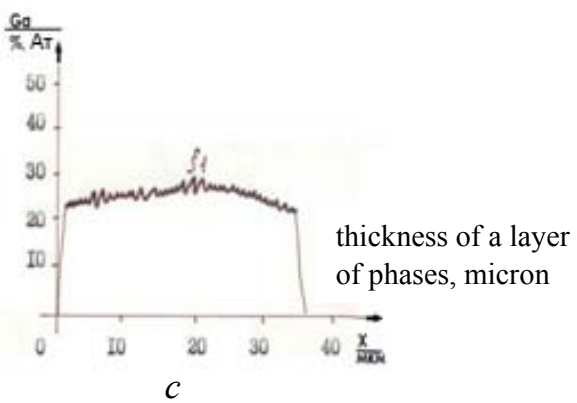

Рис. 3. Зависимость микроструктуры и фазового состава паяных швов меди галлием для интерметаллических фаз $\gamma_{1}, \gamma_{2}, \gamma_{3}$ и $\xi_{1}$ при одинаковых температуре $\left(T=400^{\circ} \mathrm{C}\right)$ и времени выдержки $\left(\tau_{\text {от. }}=60\right.$ мин $)$ изотермического отжига от толщины исходного количества галлия: a) $\mathrm{H}_{\mathrm{Ga}}=18 . .20$ мкм; $\left.b\right) \mathrm{H}_{\mathrm{Ga}}=8 \ldots 10$ мкм; $c$ ) $\mathrm{H}_{\mathrm{Ga}}=4 \ldots 5$ мкм

[Fig. 3. Dependence of a microstructure and phase structure of soldered seams of copper gallium for intermetallic phases $\gamma_{1}, \gamma_{2}, \gamma_{3}$ and $\xi_{1}$ at identical to temperature $\left(T=400^{\circ} \mathrm{C}\right)$ and hold time $\left(\tau_{\text {ог. }}=60 \mathrm{~min}\right)$ isothermal annealing from thickness of initial amount of gallium: $a) \mathrm{NGA}=18 \ldots 20 \mu \mathrm{m} ; b) \mathrm{NGA}=8 \ldots 10 \mu \mathrm{m} ; c) \mathrm{NGA}=4 \ldots 5 \mu \mathrm{m}]$ 


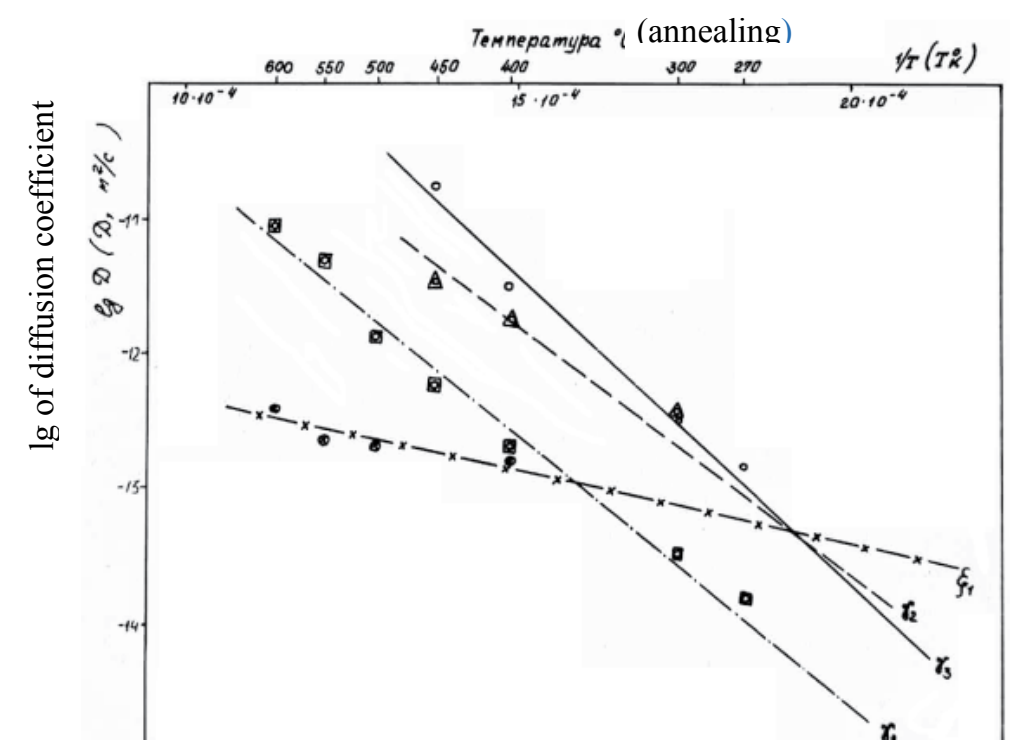

a

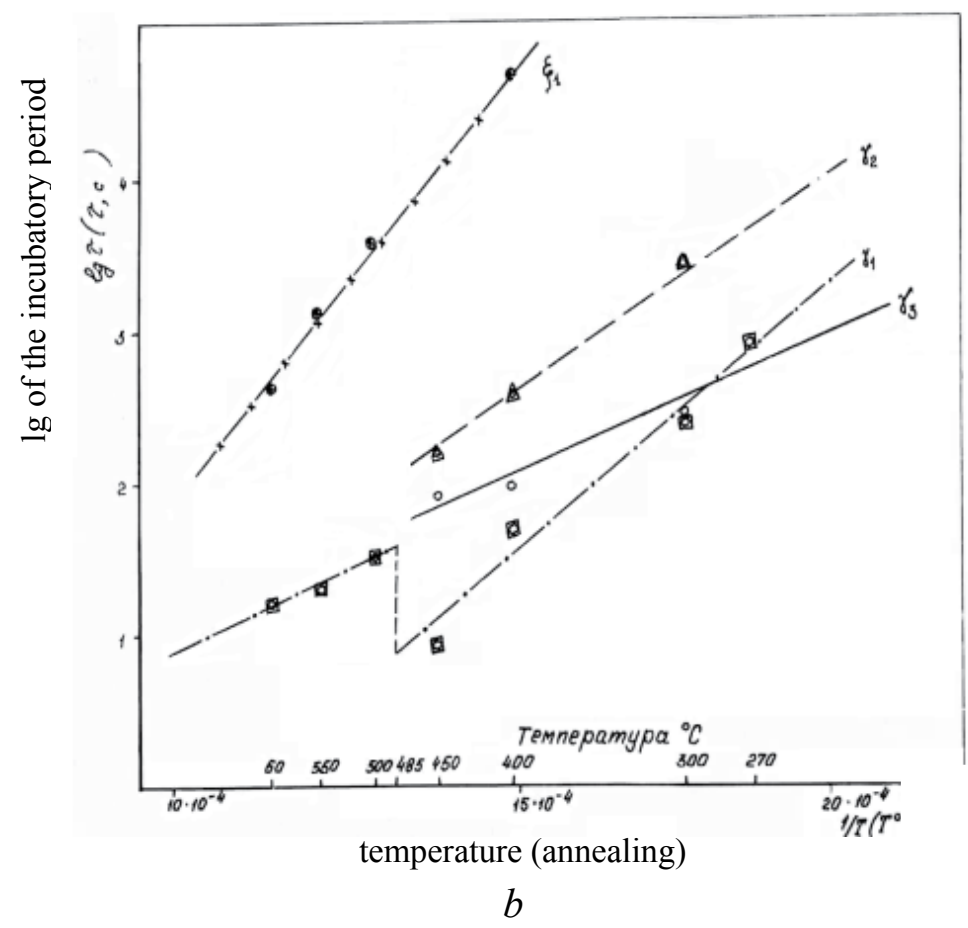

Рис. 4. Температурная зависимость коэффициентов диффузии (a) и инкубационных периодов $(b)$ для интерметаллических фаз $\gamma_{1}, \gamma_{2}, \gamma_{3}$ и $\xi_{1}$

[Fig. 4. Temperature dependence of coefficients of diffusion $(a)$ and the incubatory periods $(b)$ for intermetallic phases $\gamma_{1}, \gamma_{2}, \gamma_{3}$ and $\left.\xi_{1}\right]$

мером $15 \times 15 \times 2$ мм. Поверхность образцов подготавливали методом алмазного точения (этот метод использовался при изготовлении деталей теплообменника). На первом этапе изучали взаимодействие в системе «медь-галлий» при неограниченном поступлении компонентов. Толщина слоя галлия равнялась 100 мкм и фиксировалась закладкой полосок слюды между медными пластинами. Изо- термический отжиг проводили в вакуумной термопечи при давлении $10^{-1}$ атм. Время нагрева до $500{ }^{\circ} \mathrm{C}$ составляло 3-5 мин, время охлаждения около 10 мин. Длительность выдержки колебалась от 15 до 90 мин. После проведения металлографических исследований и рентгеноспектрального анализа был проведен расчет параметров диффузии для фаз $\gamma_{1}, \gamma_{2}, \gamma_{3}$ и $\xi_{1}$ системы «медь-галлий». 


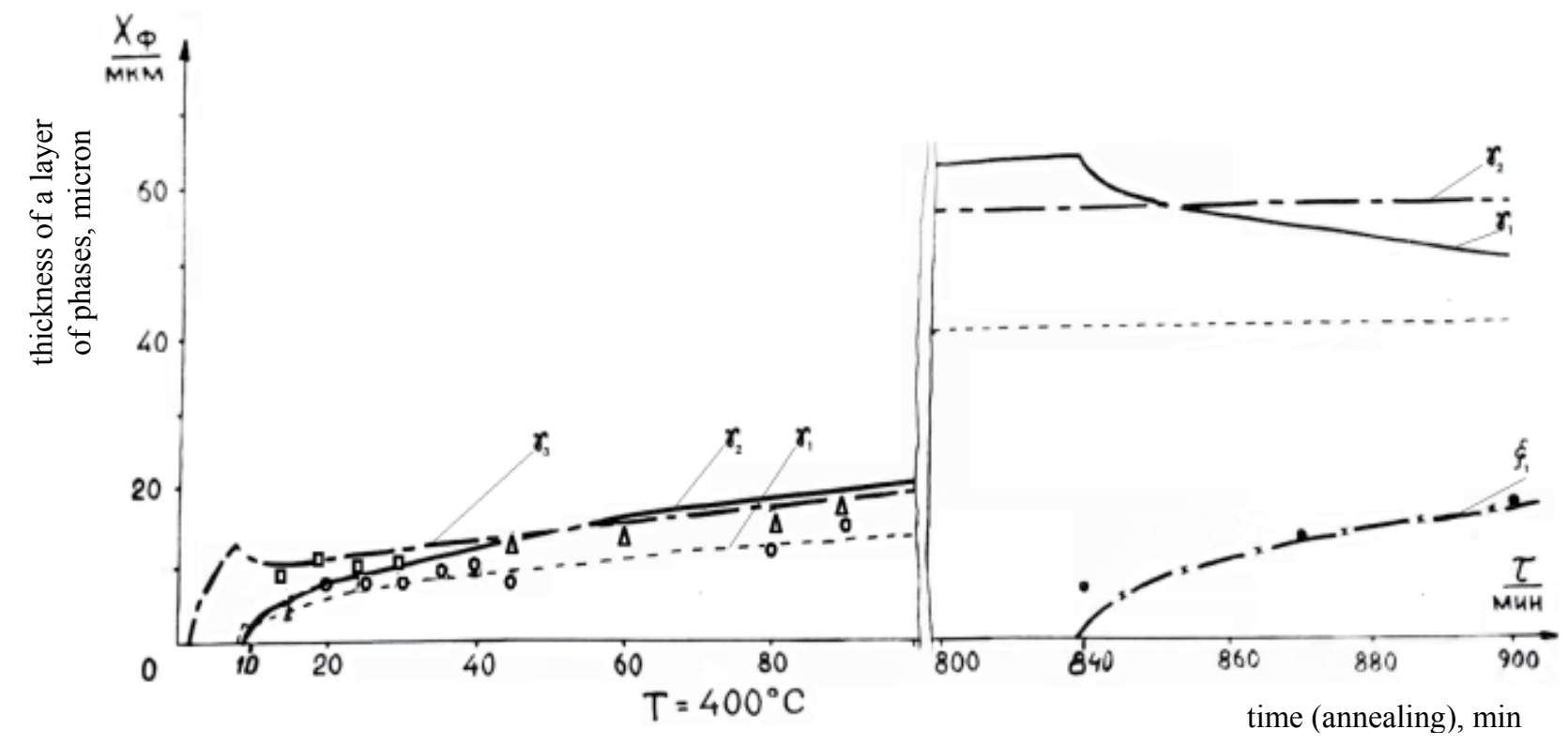

Рис. 5. Пример расчёта толщины слоев интерметаллических фаз $\gamma_{1}, \gamma_{2}, \gamma_{3}$ и $\xi_{1}$ при неограниченном поступлении галлия и разной длительности термического отжига при $T=400{ }^{\circ} \mathrm{C}$

[Fig. 5. An example of calculation of thickness of layers of intermetallic phases $\gamma_{1}, \gamma_{2}, \gamma_{3}$ and $\xi_{1}$ at unlimited intake of gallium and different duration of thermal annealing at $\left.T=400{ }^{\circ} \mathrm{C}\right]$

Для проведения расчетов при рентгеноспектральном анализе было определено распределение концентраций компонентов во всех интерметаллических слоях.

В соответствии с полученными результатами был проведен повторный расчёт диффузионных зон при неограниченном поступлении галлия для различных режимов изотермического отжига, при которых не проводились эксперименты. При этом было получено хорошее совпадение расчётных и экспериментальных данных (рис. 5 ).

Второй этап исследования включал гипотетические расчёты диффузионных зон паяных швов и интерметаллических покрытий при малых толщинах галлия с использованием параметров диффузии, полученных в условиях, когда галлий не истощался в процессах отжига.

После расчётов времени исчезновения жидкой фазы для различных температур и времени изотермического отжига был проведен расчёт изменения длительности инкубационного периода для фаз системы «медь-галлий», в частности, для фазы $\xi_{1}$ (рис. 6 ).

\section{РЕЗУЛЬТАТЫ И ИХ ОБСУЖДЕНИЕ}

Расчёт протяженности диффузионной зоны и ее фазового состава от толщины слоя галлия для паяных швов и диффузионных покрытий от температуры отжига был проведён для разных темпера-

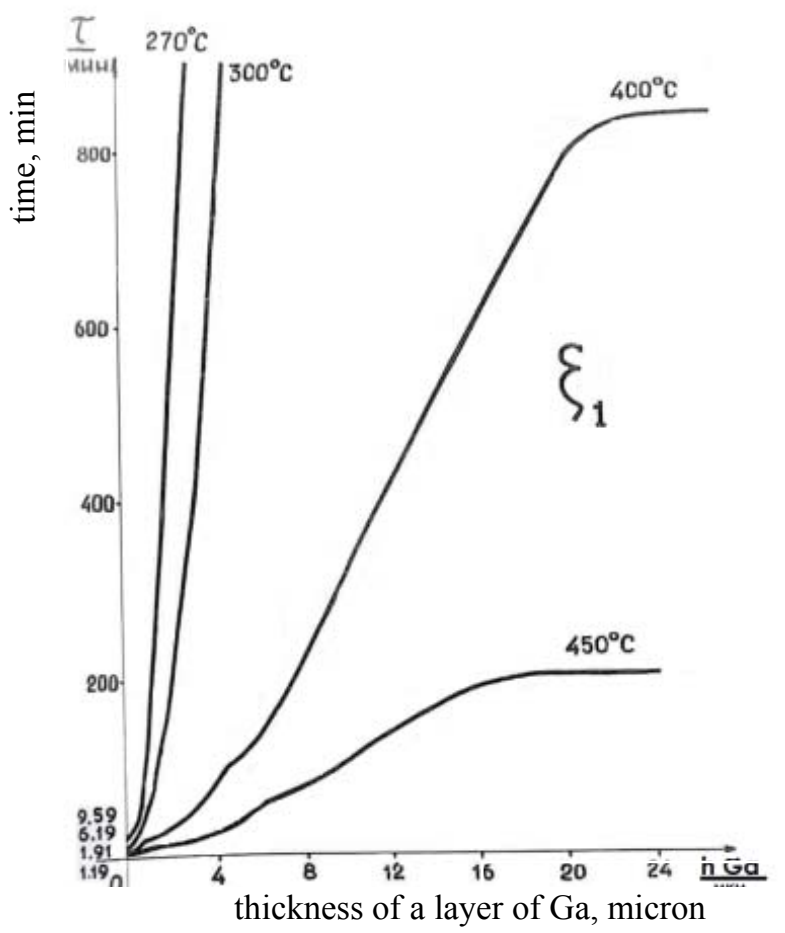

Рис. 6. Расчётная зависимость инкубационного периода интерметаллической фазы $\xi_{1}$ в системе «медь-галлий» от толщины слоя галлия для разных температур

[Fig. 6. The calculated dependence of the incubatory period of the intermetallic phase $\xi_{1}$ in the copper-gallium system from gallium layer thickness for different temperatures] 


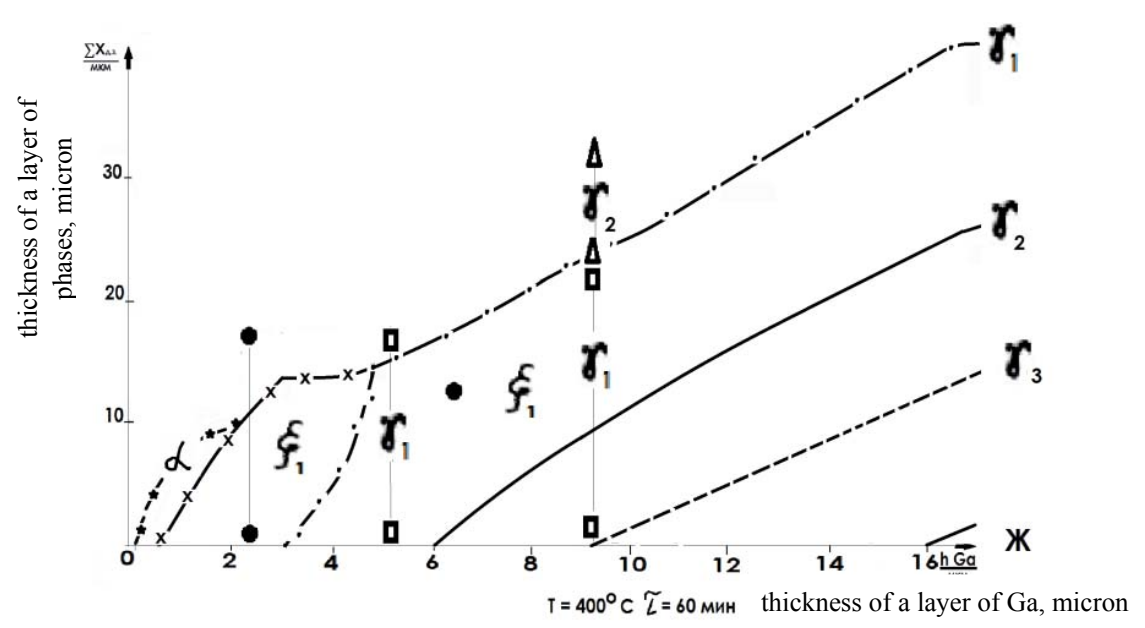

Рис. 7. Пример расчёта протяженности диффузионной зоны и ее фазового состава (для интерметаллических фаз $\gamma_{1}, \gamma_{2}, \gamma_{3}$ и $\xi_{1}$ ) от толщины слоя галлия для паяных швов и покрытий при разных температурно-временных режимах изотермического отжига (расчёт произведен для $S$ толщины диффузионной зоны паяного шва)

[Fig. 7. An example of calculation of extent of a diffusive zone and its phase structure (for intermetallic phases $\gamma_{1}, \gamma_{2}, \gamma_{3}$ and $\xi_{1}$ ) from gallium layer thickness for soldered seams and coverings at different temperature and time modes of isothermal annealing (calculation is made for $S$ thickness of a diffusive zone of the soldered seam)]

турно-временных режимов. На рис. 7 показан результат расчетов для времени отжига 60 мин при температуре $400{ }^{\circ} \mathrm{C}$.

Экспериментальные данные, нанесенные на график зависимости толщины слоя фаз от толщины слоя галлия при $T_{\text {отж }}=400{ }^{\circ} \mathrm{C}$ и $\tau_{\text {от }}=60$ мин, показали хорошее совпадение расчетных и экспериментальных данных.

На третьем этапе были проведены дополнительные эксперименты при толщинах слоя галлия от 3-4 до 18-20 мкм. При этом толщину галлия задавали созданием давления на образцы. После удаления излишков галлия и снятия давления, изотермический отжиг проводили в вакуумной термопечи при давлении $10^{-1}$ атм.

На расчётные кривые наносили экспериментальные данные. Расчёт протяженности диффузионной зоны и ее фазового состава от толщины слоя галлия для паяных швов и диффузионных покрытий от температуры отжига (рис. 7) показал хорошее совпадение с экспериментальными данными.

По результатам расчётов, представленных на рис. 4-7, в дальнейшем, были определены технологические параметры пайки теплообменников, нанесения на их поверхность интерметаллических покрытий и изготовлены несколько изделий.

Сравнительный анализ данных, проведённый по литературным источникам, посвящённым исследованию диффузии в системе «медь-галлий», показал, что по данной тематике имеется значительное количество работ, например [17-20], но все они связаны с исследованием низкотемпературных галлиевых паст-припоев. В частности, в этих работах взаимодействие галлия с дисперсным медным порошком, как правило, исследовано при температурах $<250{ }^{\circ} \mathrm{C}$. В работе [19] (без учета влияния на процесс данного взаимодействия сферичности медных порошков [10] и длительности инкубационного периода $[1,2])$ расчётным путём получен коэффициент диффузии для $\Omega$-фазы:

$$
D=1,34 \cdot 10^{-2} \cdot \exp \left(-\frac{10300 \pm 200}{2 k T}\right) \mathrm{cm}^{2} / \mathrm{c} .
$$

Инкубационные периоды для фаз $\gamma_{1}, \gamma_{2}, \gamma_{3}$ и $\xi_{1}$ при температурах $130-200{ }^{\circ} \mathrm{C}$ составляют от 5 до 500 часов. При этом при уменьшении толщины прослойки жидкого галлия инкубационный период $\xi_{1}$-фазы уменьшается. Количественной оценки параметров диффузии для фаз $\gamma_{1}, \gamma_{2}, \gamma_{3}$ и $\xi_{1}$ автор работы [19] не проводил.

\section{ЗАКЛЮЧЕНИЕ}

Решена задача об изменении длительности инкубационных периодов для фаз, которые должны появиться в диффузионной зоне после истощения слоя компонента с меньшей температурой плавления для гипотетических двухкомпонентных многофазных систем. По известным параметрам диффузии, полученным при исследовании двухкомпо- 
нентной многофазной системы при неограниченном поступлении компонентов, рассчитаны критическая толщина слоя компонента А, вызывающая это изменение, изменение длительности инкубационных периодов для разных ситуаций, фазовый состав и протяженность диффузионной зоны взаимодействующих компонентов А и В и определены технологические параметры, например, пайки теплообменников и нанесения на их поверхность интерметаллических покрытий.

\section{СПИСОК ЛИТЕРАТУРЫ}

1. Молохина Л. А., Рогалин В. Е., Филин С. А., Каплунов И. А. // Журнал физическойхимии, 2017, т. 91, № 9, c. 1468-1475. DOI: https://doi.org/10.7868/ S0044453717090242

2. Молохина Л. А., Рогалин В. Е., Филин С. А., Каплунов И. А. // Физика и химия обработки материалов. 1986, № 1, p. 123.

3. Прокошкин Д. А. Химические и термические методы обработки стали. М.;-Л.:, ОНТП, 1938, с. 343.

4. Лариков Л. Н., Рябов В. Р., Фальченко В. М. Диффузионные проиессы в твердой фазе при сварке. М: Машиностроение, 1975, 192 с.

5. Бокштейн Б. С. Диффузия в металлах. М: Металлургия, 1978, 248 с. 1198.

6. Ширинян А. С. // ФTT, 2010 , т. 52 , вып. 6, с. $1191-$

7. Рохлин Э. А. Сварка. Л.: Судостроение, Ленингр. отд. 1968, вып. 11, с. 99-107.

8. Гуревич Л. М., Трыков Ю. П., Арисова В. Н. и др. // Известия ВолГТУ,. 2009, № 11 (59), Серия «Про- блемы материаловедения сварки и прочности в машиностроении», с. 5-11.

9. Шморгун В. Г., Трыков Ю. П., Слаутин О. В., Богданов А. И., Битюцких А. Е. // Известия ВолГТУ, 2009, № 11 (59), Серия «Проблемы материаловедения сварки и прочности в машиностроении», с. 15-19.

10. Долгов Ю. С., Сидохин Ю. Ф. Вопросы формирования паяного шва. М.: Машиностроение, 1973, $138 \mathrm{c}$.

11. Лашко Н. Ф., Лашко С. В. Вопросы теории и технологии пайки. Саратов, Из-во Саратовского университета, 1976, 248 c.

12. Рябов В. Р. Сварка алюминия и его сплавов с другими металлами. Киев, Наукова думка, 1983, 264 с.

13. Лашко Н. Ф., Лашко С. В. Пайка металлов. М.: Машиностроение, 1988, 376 с.

14. Рослякова Л. И., Росляков И. Н. // Упрочняющие технологии и покрытия. 2014, № 4 (112), с. 32.

15. Иванов С. Г., Гурьев М. А., Гурьев А. М. // АКтуальные проблемы в машиностроении, 2015, № 2, c. $416-420$.

16. Гусак А. М. Дис... канд. физ-мат. наук. Москва, $1982,186 \mathrm{c}$.

17. Мерер Х. Диффузия в твердых телах / Пер. с англ. Долгопрудный, Интеллект, 2011, 536 с.

18. Тихомирова О. И. Дис. ... канд. техн. наук. Москва, 1969, с. 176.

19. Казаков В. С. Дис. ... канд. техн. наук. Красноярск, 2007. URL: http://www.dissercat.com/content/razrabotka-gallievykh-past-pripoev-dlya-nizkotemperaturnoipaiki-mednykh-i-titanovykh-splavo

20. Чуларис А. А., Михайлова М. М., Чумаченко Г. В. // Вестник ДГТУ. 2010, т. 10, № 2(45), с. 183-188. URL: https://vestnik.donstu.ru/jour/article/view/961/956

\title{
FEATURES OF CALCULATION OF PARAMETERS OF DIFFUSION IN TWO-COMPONENT MULTIPHASE SYSTEMS AT "LIMITED" INTAKE OF THE COMPONENT WITH A SMALLER TEMPERATURE OF MELTING
}

\author{
(C) 2018 L. A. Molokhina1, S. A. Filin² \\ ${ }^{1}$ Kibalchich str., 2, corpus 4, apartment 86, 129164 Moscow, Russia \\ e-mail: lara.molokhina@mail.ru \\ ${ }^{2}$ Plekhanov Russian University of Economics, 36, Stremynny Lane, 117997 Moscow, Russia \\ e-mail: Filin.SA@rea.ru
}

Received 31.08.2018

\begin{abstract}
Subject of research - process of the solution of a task on change of duration of the incubatory periods for phases which have to appear in a diffusive zone after the component layer with a smaller temperature of melting is exhausted, topic - identification of features of calculation of parameters of diffusion in two-component multiphase systems at "limited" intake of this component, the purpose
\end{abstract}


- to offer a way of the solution of a task on change of duration duration of the incubatory periods for phases which have to appear in a diffusive zone after the component layer with a smaller temperature of melting is exhausted.

Methods and methodology. Chemical and thermal methods of processing, in particular, method of diamond turning; a method of the smallest squares in a method of calculation of parameters of diffusion in two-component multiphase system.

Results. The method of calculation of parameters of diffusion in two-component multiphase systems at "limited" intake of a component with a smaller temperature of melting is offered. The dependence of thickness of a layer of this component in two-component multiphase system is constructed. Dynamics of growth of phases in this system from diffusion time is investigated. The dependence of the incubatory period of appearance of the subsequent phases from layer thickness of component with a smaller temperature of melting in two-component multiphase system. The example of the practical solution of a task on change of duration of the incubatory periods for phases in the coppergallium system is given which have to appear in a diffusive zone after the component layer with a smaller temperature of melting is exhausted.

Conclusions. It is shown that in two-component multiphase system for each subsequent new phase there is a critical value of thickness of a layer of a component with a smaller temperature of melting. At a thickness, smaller critical value, a certain duration of process of annealing causes change of the incubatory period of these phases regardless of the fact that preceded it: component exhaustion with a smaller temperature of melting or dissolution of one of the previous phases.

Keywords: diffusion, phase, interphase border, multiphase system, isothermal annealing, mathematical model, component.

DOI: https://doi.org/10.17308/kcmf.2018.20/637

\section{REFERENCES}

1. Molokhina L. A., Rogalin V. E., Kaplunov I. A., Filin S. A. Russian Journal of Physical Chemistry A, 2017, vol. 91, no. 9, pp. 1635-1641. DOI: https://doi.org/10.1134/ S0036024417090217

2. Molohina L. A., Rogalin V. E., Filin S. A., Kaplunov I. A. Fizika i himiya obrabotki materialov, 1986, no. 1, p. 123. (in Russ.)

3. Prokoshkin D. A. Himicheskie i termicheskie metody obrabotki stali [Chemical and Thermal Methods of Steel Working]. Moscow-Leningrad, ONTP Publ., 1938, pp. 3-43. (in Russ.)

4. Larikov L. N., Ryabov V. R., Fal'chenko V. M. Diffuzionnye processy $v$ tverdoj faze pri svarke [Diffusive Processes in a Firm Phase When Welding]. Moscow, Mashinostroenie Publ., 1975, 192 p. (in Russ.)

5. Bokstein B. S. Diffuziya v metallah [Diffusion in Metals]. Moscow, Metallurgy Publ., 1978, 248 p. (in Russ.)

6. Shirinyan A. S. Fizika tverdogo tela [Physics of the Solid State]. 2010, vol. 52, no. 6, pp. pp 1267-1275. DOI: https://doi.org/10.1134/S1063783410060235

7. Rohlin 'E. A. Svarka [Welding]. Leningrad, Sudostroenie. Leningr. otd. Publ., 1968, no. 11, pp. 99-107. (in Russ.)

8. Gurevich L. M., Trykov Yu. P., Arisova V. N., et al. Izvestia VSTU, 2009, no. 11 (59), Seriya «Problemy materialovedeniya svarki i prochnosti $\mathrm{v}$ mashinostroenii», pp. 5-11. (in Russ.)
9. Shmorgun V. G., Trykov Yu. P., Slautin O. V., Bogdanov A. I., Bityutskikh A. E. Izvestia VSTU, 2009, no. 11 (59), Seriya «Problemy materialovedeniya svarki i prochnosti v mashinostroenii», pp. 15-19. (in Russ.)

10. Dolgov Yu. S., Sidohin Yu. F. Voprosy formirovaniya payanogo shva [Questions of Formation of a Soldered Seam]. Moscow, Mashinostroenie Publ., 1973, 138 p. (in Russ.)

11. Lashko N. F., Lashko S. V. Voprosy teorii i tehnologii pajki [Questions of the Theory and Technology of Soldering]. Saratov, Iz-vo Saratovskogo universiteta Publ., 1976, 248 p. (in Russ.)

12. Ryabov V. R. Svarka alyuminiya i ego splavov s drugimi metallami [Welding of Aluminum and its Alloys With other Metals]. Kiev, Naukova dumka Publ., 1983, 264 p. (in Russ.)

13. Lashko N. F., Lashko S. V. Pajka metallov [Soldering of Metals]. Moscow, Mashinostroenie Publ., 1988, 376 p. (in Russ.)

14. Roslyakova L. I., Roslyakov I. N. Uprochnyayuschie tehnologii i pokrytiya, 2014, no. 4 (112), p. 32. (in Russ.)

15. Ivanov S. G., Gur'ev M. A., Gur'ev A. M. Aktual'nye problemy v mashinostroenii, 2015, no. 2, pp. 416-420. (in Russ.)

16. Gusak A. M. Dis. kand. fiz-mat nauk. Moscow, 1982, 186 p. (in Russ.)

17. Mehrer H. Diffusion in Solids. Springer. 2007. $645 \mathrm{p}$.

18. Tihomirova O. I . Dis. kand. tehn. nauk. Moscow, 1969, p. 176. (in Russ.) 
19. Kazakov V. S. Dis. kand. tehn. nauk. Krasnoyarsk, 2007. URL: http://www.dissercat.com/content/razrabotkagallievykh-past-pripoev-dlya-nizkotemperaturnoi-paikimednykh-i-titanovykh-splavo. (in Russ.)
20. Chularis A. A., Mihajlova M. M., Chumachenko G. V. Vestnik DGTU [Vestnik of Don State Technical University], 2010, vol. 10, no. 2(45), pp. 183-188. Available at: https://vestnik.donstu.ru/jour/article/view/961/956 (in Russ.)
Молохина Лариса Аркадьевна - к. т. н., ул. Кибальчича, д. 2 , корп. 4 , кв. 86, Москва, Россия; тел.: +7 (985) 9962052, e-mail: lara.molokhina@mail.ru

Филин Сергей Александрович - д. э. н., к. т. н., доцент, профессор, Российский экономический университет им. Г. В. Плеханова, Москва, Россия; тел.: +7 (926) 8122737, e-mail: Filin.SA@rea.ru
Larisa A. Molokhina-Cand. Sci. (Eng.), Kibalchich str., 2, corpus 4, apartment 86, Moscow, Russia; tel.: +7 (985) 9962052, e-mail: lara.molokhina@mail.ru

Sergey A. Filin-Dr. Sci. (Econ.), Cand. Sci. (Eng.), Associate Professor, Professor, Plekhanov Russian University of Economics, Moscow, Russia; tel.: +7 (926) 8122737, e-mail: Filin.SA@rea.ru 